Stud. Univ. Babeş-Bolyai Math. 65(2020), No. 4, 503-519

DOI: 10.24193 /subbmath.2020.4.03

\title{
On oscillatory second order nonlinear impulsive systems of neutral type
}

\author{
Arun Kumar Tripathy and Shyam Sundar Santra
}

\begin{abstract}
In this work, the necessary and sufficient conditions for oscillation of a class of second order neutral impulsive systems are established and our impulse satisfies a discrete neutral nonlinear equation of similar type. Further, one illustrative example showing applicability of the new result is included.
\end{abstract}

Mathematics Subject Classification (2010): 34C10, 35K40, 34K11.

Keywords: Oscillation, nonoscillation, neutral, delay, non-linear, Lebesgue's dominated convergence theorem, Banach's fixed point theorem.

\section{Introduction}

Impulsive differential equations are now recognized as an excellent source of models to simulate processes and phenomena observed in control theory, physics, chemistry, population dynamics, industrial robotics, biotechnologies, economics and to mention a few. Due to the wide range application of this theory to the real world problem, a good number of interests has been given to study impulsive differential equations, since it is much richer than the corresponding theory of differential equations without impulse effect. We refer the readers to the monographs $[1,2,10,13,14]$ and [18], where a number of properties of their solutions are discussed and the references cited there in.

In [28], Tripathy has considered the impulsive system

$$
\left(E_{1}\right)\left\{\begin{array}{l}
(y(t)+p(t) y(t-\tau))^{\prime}+q(t) G(y(t-\sigma))=0, t \neq \tau_{k}, k \in \mathbb{N}, \\
\Delta\left(y\left(\tau_{k}\right)+p\left(\tau_{k}\right) y\left(\tau_{k}-\tau\right)\right)+q\left(\tau_{k}\right) G\left(y\left(\tau_{k}-\sigma\right)\right)=0, k \in \mathbb{N},
\end{array}\right.
$$

and studied the oscillatory character of solutions of the system. For all ranges of $p(t)$, he has established the oscillation criteria for the impulsive system $\left(E_{1}\right)$ which is highly nonlinear and $G$ could be linear, sublinear or superlinear. In [29], Tripathy

This work is supported by the Department of Science and Technology (DST), New Delhi, India, through the bank instruction order No. DST/INSPIRE Fellowship/2014/140, dated Sept. 15, 2014. 
and Santra have made an attempt to establish the necessary and sufficient condition for oscillation of a class of forced impulsive differential equations of the form

$$
\left\{\begin{array}{l}
(y(t)+p(t) y(t-\tau))^{\prime}+q(t) G(y(t-\sigma))=f(t), \quad t \neq \tau_{k}, k \in \mathbb{N} \\
\Delta\left(y\left(\tau_{k}\right)+p\left(\tau_{k}\right) y\left(\tau_{k}-\tau\right)\right)+r\left(\tau_{k}\right) G\left(y\left(\tau_{k}-\sigma\right)\right)=g\left(\tau_{k}\right), \quad k \in \mathbb{N} .
\end{array}\right.
$$

In an another paper [30], Tripathy and Santra have studied the characterization of the impulsive system

$$
\left(E_{2}\right)\left\{\begin{array}{l}
(y(t)-r y(t-\tau))^{\prime}+q y(t-\sigma)=0, t \neq \tau_{k}, k \in \mathbb{N}, \\
\Delta\left(y\left(\tau_{k}\right)-r y\left(\tau_{k}-\tau\right)\right)+p y\left(\tau_{k}-\sigma\right)=0, k \in \mathbb{N},
\end{array}\right.
$$

and linearized oscillation of the system

$$
\left(E_{3}\right)\left\{\begin{array}{l}
(y(t)-r(t) g(y(t-\tau)))^{\prime}+q(t) f(y(t-\sigma))=0, t \neq \tau_{k}, k \in \mathbb{N}, \\
\Delta\left(y\left(\tau_{k}\right)-r\left(\tau_{k}\right) g\left(y\left(\tau_{k}-\tau\right)\right)\right)+p\left(\tau_{k}\right) f\left(y\left(\tau_{k}-\sigma\right)\right)=0, \quad k \in \mathbb{N} .
\end{array}\right.
$$

They have established the conditions pertaining the oscillation of the system $\left(E_{2}\right)$ using the pulsatile constant and hence the linearized oscillation results carried out for $\left(E_{3}\right)$ by using its limiting equation $\left(E_{2}\right)$.

Motivated by the works $[28,29,30]$, an attempt is made here to discuss the oscillation properties of a class of second order neutral impulsive system of the form:

$$
(E)\left\{\begin{array}{l}
\left(r(t)(y(t)+p(t) y(t-\tau))^{\prime}\right)^{\prime}+q(t) G(y(t-\sigma))=0, \quad t \neq \tau_{k}, k \in \mathbb{N}, \\
\Delta\left(r\left(\tau_{k}\right)\left(y\left(\tau_{k}\right)+p\left(\tau_{k}\right) y\left(\tau_{k}-\tau\right)\right)^{\prime}\right)+q\left(\tau_{k}\right) G\left(y\left(\tau_{k}-\sigma\right)\right)=0, \quad k \in \mathbb{N},
\end{array}\right.
$$

where $\tau, \sigma \in \mathbb{R}_{+}=(0,+\infty) ; \tau_{1}, \tau_{2}, \cdots, \tau_{k}, \cdots$ are the fixed moments of impulse effect; $p\left(\tau_{k}\right), r\left(\tau_{k}\right)$ and $q\left(\tau_{k}\right)$ are real sequences for $k \in \mathbb{N} ; G \in C(\mathbb{R}, \mathbb{R})$ is nondecreasing such that $x G(x)>0$ for $x \neq 0 ; q, r \in C\left(\mathbb{R}_{+}, \mathbb{R}_{+}\right) ; p \in P C\left(\mathbb{R}_{+}, \mathbb{R}\right)$, and

$$
\begin{aligned}
& \Delta\left(r\left(\tau_{k}\right) z^{\prime}\left(\tau_{k}\right)\right)=r\left(\tau_{k}+0\right) z^{\prime}\left(\tau_{k}+0\right)-r\left(\tau_{k}-0\right) z^{\prime}\left(\tau_{k}-0\right) ; \\
& y\left(\tau_{k}-0\right)=y\left(\tau_{k}\right) \quad \text { and } \quad y\left(\tau_{k}-\tau-0\right)=y\left(\tau_{k}-\tau\right), \quad k \in \mathbb{N} .
\end{aligned}
$$

The objective of this work is to establish the necessary and sufficient conditions for oscillation of the impulsive system $(E)$. Here, we are concerned with oscillating systems which remain oscillating after being perturbed by instantaneous change of state. We may note that this type of work is very rare in the literature signifying that the impulse of the differential equation follows a difference equation of same type. In this direction, we refer the reader to some of the related works $[3,4,5,6,7,8,9,11$, $12,15,16,17,19,26,27,32,33,34]$ and the references cited there in.

Definition 1.1. A function $y:[-\rho,+\infty) \rightarrow \mathbb{R}$ is said to be a solution of $(E)$ with initial function $\phi \in C([-\rho, 0], \mathbb{R})$, if $y(t)=\phi(t)$ for $t \in[-\rho, 0], y \in P C\left(\mathbb{R}_{+}, \mathbb{R}\right)$, $z(t)=y(t)+p(t) y(t-\tau)$ and $r(t) z^{\prime}(t)$ are continuously differentiable for $t \in \mathbb{R}_{+}$, and $y(t)$ satisfies $(E)$ for all sufficiently large $t \geq 0$, where $\rho=\max \{\tau, \sigma\}, P C\left(\mathbb{R}_{+}, \mathbb{R}\right)$ is the set of all functions $U: \mathbb{R}_{+} \rightarrow \mathbb{R}$ which are continuous for $t \in \mathbb{R}_{+}, t \neq \tau_{k}, k \in \mathbb{N}$, continuous from the left- side for $t \in \mathbb{R}_{+}$, and have discontinuity of the first kind at the points $\tau_{k} \in \mathbb{R}_{+}, k \in \mathbb{N}$. 
Definition 1.2. A nontrivial solution $y(t)$ of $(E)$ is said to be nonoscillatory, if there exists a point $t_{0} \geq 0$ such that $y(t)$ has a constant sign for $t \geq t_{0}$. Otherwise, the solution $y(t)$ is said to be oscillatory.

Definition 1.3. A solution $y(t)$ of $(E)$ is said to be regular, if it is defined on some interval $\left[T_{y},+\infty\right) \subset\left[t_{0},+\infty\right)$ and

$$
\sup \left\{|y(t)|: t \geq T_{y}\right\}>0
$$

for every $T_{y} \geq T$. A regular solution $y(t)$ of $\left(E_{1}\right)$ is said to be eventually positive (eventually negative), if there exists $t_{1}>0$ such that $y(t)>0(y(t)<0)$ for $t \geq t_{1}$.

\section{Main results}

This section deals with the necessary and sufficient conditions for oscillation of all solutions of the impulsive system $(E)$. We introduce the following assumptions for our use in the sequel:

$\left(A_{0}\right) \int_{0}^{\infty} \frac{d t}{r(t)}<\infty$ if and only if $\sum_{k=1}^{\infty} \frac{1}{r\left(\tau_{k}\right)}<\infty$;

$\left(A_{1}\right) 0<\tau_{1}<\tau_{2}<\cdots$ and $\lim _{k \rightarrow \infty} \tau_{k}=+\infty$;

$\left(A_{2}\right) p \in P C\left(\mathbb{R}_{+}, \mathbb{R}\right), p_{k}=p\left(\tau_{k}-0\right)=p\left(\tau_{k}\right), r_{k}=r\left(\tau_{k}-0\right)=r\left(\tau_{k}\right)$ and $q_{k}=q\left(\tau_{k}-0\right)=q\left(\tau_{k}\right), k \in \mathbb{N}$.

Theorem 2.1. Let $-1<-a \leq p(t) \leq 0, a>0$ and $t \in \mathbb{R}_{+}$. Assume that $\left(A_{0}\right),\left(A_{1}\right)$ and $\left(A_{2}\right)$ hold. Furthermore, assume that

$\left(A_{3}\right) G(-u)=-G(u), u \in \mathbb{R}$

and

$\left(A_{4}\right) \int_{\sigma}^{\infty} q(t) G(C R(t-\sigma)) d t+\sum_{k=1}^{\infty} q\left(\tau_{k}\right) G\left(C R\left(\tau_{k}-\sigma\right)\right)<+\infty$ for every constant $C>0$

hold, where $R(t)=\int_{0}^{t} \frac{d s}{r(s)}$. Then every unbounded solution of the system $(E)$ oscillates if and only if

$\left(A_{5}\right) \int_{0}^{\infty} \frac{d s}{r(s)}<+\infty$.

Proof. Let $y(t)$ be a regular solution of $(E)$ which is unbounded. So, there exists $t_{0}>0$ such that $y(t)>0$ or $<0$, for $t \geq t_{0}$. Without loss of generality and because of $\left(A_{3}\right)$, we may assume that $y(t)>0, \quad y(t-\tau)>0$ and $y(t-\sigma)>0$, for $t \geq t_{1}>t_{0}+\rho$. Setting

$$
z(t)=y(t)+p(t) y(t-\tau)
$$

in the system $(E)$, it follows that

$$
\begin{aligned}
\left(r(t) z^{\prime}(t)\right)^{\prime} & =-q(t) G(y(t-\sigma))<0, \quad t \neq \tau_{k} \\
\Delta\left(r\left(\tau_{k}\right) z^{\prime}\left(\tau_{k}\right)\right) & =-q_{k} G\left(y\left(\tau_{k}-\sigma\right)\right)<0, \quad k \in \mathbb{N}
\end{aligned}
$$

for $t \geq t_{1}$. Hence, there exists $t_{2}>t_{1}$ such that $r(t) z^{\prime}(t)$ is nonincreasing on $\left[t_{2}, \infty\right)$. Since $z(t)$ is monotonic, then there exists $t_{3}>t_{2}$ such that $z(t)>0$ or $<0$, for 
$t \geq t_{3}$. Indeed, $z(t)<0$ for $t \geq t_{3}$ implies that $y(t)<y(t-\tau), y\left(\tau_{k}\right)<y\left(\tau_{k}-\tau\right)$, $y\left(\tau_{k}+0\right)<y\left(\tau_{k}+0-\tau\right)$ and hence

$$
\begin{aligned}
& y(t)<y(t-\tau)<y(t-2 \tau)<\cdots<y\left(t_{3}\right), \quad t \neq \tau_{k}, \\
& y\left(\tau_{k}\right)<y\left(\tau_{k}-\tau\right)<y\left(\tau_{k}-2 \tau\right)<\cdots<y\left(t_{3}\right), \quad k \in \mathbb{N}, \\
& y\left(\tau_{k}+0\right)<y\left(\tau_{k}+0-\tau\right)<y\left(\tau_{k}+0-2 \tau\right)<\cdots<y\left(t_{3}\right), \quad k \in \mathbb{N},
\end{aligned}
$$

that is, $y(t)$ is bounded, which is absurd. Hence, $z(t)>0$ for $t \geq t_{3}$. If $r(t) z^{\prime}(t)>0$ for $t \geq t_{3}$, then $r(t) z^{\prime}(t)$ is nonincreasing on $\left[t_{3}, \infty\right)$ and hence there exist a constant $C>0$ and $t_{4}>t_{3}$ such that $r(t) z^{\prime}(t) \leq C$ for $t \geq t_{4}$. Consequently,

$$
z(t) \leq z\left(t_{4}\right)+\sum_{t_{4} \leq \tau_{k}<t} z^{\prime}\left(\tau_{k}\right)+C \int_{t_{4}}^{t} \frac{d s}{r(s)},
$$

since $r\left(\tau_{k}\right) z^{\prime}\left(\tau_{k}\right) \leq C$. Therefore, the last inequality becomes

$$
z(t) \leq z\left(t_{4}\right)+C\left[\int_{t_{4}}^{t} \frac{d s}{r(s)}+\sum_{t_{4} \leq \tau_{k}<t} \frac{1}{r\left(\tau_{k}\right)}\right]<\infty
$$

as $t \rightarrow \infty$ due to $\left(A_{0}\right)$. On the other hand, $y(t)$ is unbounded, and thus there exists $\left\{\eta_{n}\right\}$ such that $\eta_{n} \rightarrow \infty$ as $n \rightarrow \infty, y\left(\eta_{n}\right) \rightarrow \infty$ as $n \rightarrow \infty$ and

$$
y\left(\eta_{n}\right)=\max \left\{y(s): t_{3} \leq s \leq \eta_{n}\right\} .
$$

Therefore,

$$
\begin{aligned}
z\left(\eta_{n}\right) & =y\left(\eta_{n}\right)+p\left(\eta_{n}\right) y\left(\eta_{n}-\tau\right) \\
& \geq(1-a) y\left(\eta_{n}\right) \rightarrow+\infty, \quad \text { as } \quad t \rightarrow \infty
\end{aligned}
$$

implies that $z(t)$ (ultimately $z\left(\tau_{k}\right)$ for $k \in \mathbb{N}$ ) is unbounded, a contradiction.

Obviously, the case $r(t) z^{\prime}(t)<0, z(t)>0$ for $t \geq t_{3}$ is not possible.

Hence, every unbounded solution of the system $(E)$ oscillates.

Next, we suppose that $\left(A_{5}\right)$ doesn't hold. Assume that

$$
\int_{0}^{\infty} \frac{d s}{r(s)}=+\infty
$$

and due to our assumption $\left(A_{4}\right)$, let

$$
\int_{T}^{\infty} q(t) G(C R(t-\sigma)) d t+\sum_{k=1}^{\infty} q_{k} G\left(C R\left(\tau_{k}-\sigma\right)\right) \leq \frac{C}{4}, C>0 .
$$

Let's consider

$$
\begin{gathered}
M=\{y: y \in C([T-\rho,+\infty), \mathbb{R}), y(t)=0 \quad \text { for } \quad t \in[T-\rho, T] \quad \text { and } \\
\left.\frac{C}{4}[R(t)-R(T)] \leq y(t) \leq C[R(t)-R(T)]\right\}
\end{gathered}
$$


and define $\Phi: M \rightarrow C([T-\rho,+\infty), \mathbb{R})$ such that

$$
(\Phi y)(t)=\left\{\begin{array}{l}
0, \quad t \in[T-\rho, T) \\
-p(t) y(t-\tau)+\int_{T}^{t} \frac{1}{r(u)}\left[\frac{C}{4}+\int_{u}^{\infty} q(s) G(y(s-\sigma)) d s\right. \\
\left.+\sum_{k=1}^{\infty} q_{k} G\left(y\left(\tau_{k}-\sigma\right)\right)\right] d u, \quad t \geq T .
\end{array}\right.
$$

For every $y \in M$,

$$
\begin{aligned}
(\Phi y)(t) & \geq \int_{T}^{t} \frac{1}{r(u)}\left[\frac{C}{4}+\int_{u}^{\infty} q(s) G(y(s-\sigma)) d s+\sum_{k=1}^{\infty} q_{k} G\left(y\left(\tau_{k}-\sigma\right)\right)\right] d u \\
& \geq \frac{C}{4} \int_{T}^{t} \frac{d u}{r(u)}=\frac{C}{4}[R(t)-R(T)]
\end{aligned}
$$

and $y(t) \leq C R(t)$ implies that

$$
\begin{aligned}
(\Phi y)(t) & \leq-p(t) y(t-\tau)+\frac{C}{2} \int_{T}^{t} \frac{d u}{r(u)} \\
& \leq a C[R(t-\tau)-R(T)]+\frac{C}{2}[R(t)-R(T)] \\
& \leq a C[R(t)-R(T)]+\frac{C}{2}[R(t)-R(T)] \\
& =\left(a+\frac{1}{2}\right) C[R(t)-R(T)] \\
& \leq C[R(t)-R(T)]
\end{aligned}
$$

implies that $(\Phi y)(t) \in M$. Define $u_{n}:[T-\rho,+\infty) \rightarrow \mathbb{R}$ by the recursive formula

$$
u_{n}(t)=\left(\Phi u_{n-1}\right)(t), \quad n \geq 1,
$$

with the initial condition

$$
u_{0}(t)=\left\{\begin{array}{l}
0, \quad t \in[T-\rho, T) \\
\frac{C}{4}[R(t)-R(T)], \quad t \geq T .
\end{array}\right.
$$

Inductively it is easy to verify that

$$
\frac{C}{4}[R(t)-R(T)] \leq u_{n-1}(t) \leq u_{n}(t) \leq C[R(t)-R(T)] .
$$

for $t \geq T$. Therefore for $t \geq T-\rho, \lim _{n \rightarrow \infty} u_{n}(t)$ exists. Let

$$
\lim _{n \rightarrow \infty} u_{n}(t)=u(t) \text { for } t \geq T-\rho .
$$

By the Lebesgue's dominated convergence theorem $u \in M$ and $(\Phi u)(t)=u(t)$, where $u(t)$ is a solution of the impulsive system $(E)$ on $[T-\rho, \infty)$ such that $u(t)>0$. Hence, $\left(A_{5}\right)$ is necessary. This completes the proof of the theorem.

Remark 2.1. In Theorem 2.1, $G$ could be linear, sublinear or superlinear.

Theorem 2.2. Let $-1<-a \leq p(t) \leq 0$, $a>0$ for $t \in \mathbb{R}_{+}$. Assume that $\left(A_{1}\right)-\left(A_{3}\right)$ and $\left(A_{5}\right)$ hold. Furthermore, assume that

$\left(A_{6}\right) \int_{T}^{\infty} \frac{1}{r(t)}\left[\int_{T}^{t} q(s) G\left(C R_{1}(s-\sigma)\right) d s+\sum_{k=1}^{\infty} q\left(\tau_{k}\right) G\left(C R_{1}\left(\tau_{k}-\sigma\right)\right)\right] d t=+\infty$ 
and

$\left(A_{7}\right) \int_{T}^{\infty} q(s) d s+\sum_{k=1}^{\infty} q\left(\tau_{k}\right)=+\infty$

hold for every constants $C, T>0$, where $R_{1}(t)=\int_{t}^{\infty} \frac{d s}{r(s)}$. Then every solution of the system $(E)$ either oscillates or converges to zero.

Proof. Let $y(t)$ be a regular solution of $(E)$. Proceeding as in Theorem 2.1, we have (2.2) for $t \geq t_{1}$. Hence, there exists $t_{2}>t_{1}$ such that $r(t) z^{\prime}(t)$ and $z(t)$ are of constant sign on $\left[t_{2}, \infty\right)$. If $z(t)<0$ for $t \geq t_{2}$, then $y(t)$ is bounded. Consequently, $\lim _{t \rightarrow \infty} z(t)$ exists. As a result,

$$
\begin{aligned}
0 & \geq \lim _{t \rightarrow \infty} z(t)=\limsup _{t \rightarrow \infty} z(t) \\
& \geq \limsup _{t \rightarrow \infty}(y(t)-a y(t-\tau)) \\
& \geq \limsup _{t \rightarrow \infty} y(t)+\liminf _{t \rightarrow \infty}(-a y(t-\tau)) \\
& =(1-a) \limsup _{t \rightarrow \infty} y(t)
\end{aligned}
$$

implies that $\limsup _{t \rightarrow \infty} y(t)=0[\because 1-a>0]$ and thus $\lim _{t \rightarrow \infty} y(t)=0$ for $t \neq \tau_{k}$, $k \in \mathbb{N}$. We may note that $\left\{y\left(\tau_{k}-0\right)\right\}_{k \in \mathbb{N}}$ and $\left\{y\left(\tau_{k}+0\right)\right\}_{k \in \mathbb{N}}$ are sequences of reals, and because of continuity of $y$

$$
\lim _{k \rightarrow \infty} y\left(\tau_{k}-0\right)=0=\lim _{k \rightarrow \infty} y\left(\tau_{k}+0\right)
$$

due to

$$
\liminf _{t \rightarrow \infty} y(t)=0=\limsup _{t \rightarrow \infty} y(t) .
$$

Hence for all $t$ and $\tau_{k}, k \in \mathbb{N}, \lim _{t \rightarrow \infty} y(t)=0$. Let $z(t)>0$ for $t \geq t_{2}$. If $r(t) z^{\prime}(t)<0$ for $t \geq t_{2}$, then $z(t)$ is bounded and hence $\lim _{t \rightarrow \infty} z(t)$ exists. Therefore, for $s \geq t>t_{2}$, $r(s) z^{\prime}(s) \leq r(t) z^{\prime}(t)$ implies that

$$
z^{\prime}(s) \leq \frac{r(t) z^{\prime}(t)}{r(s)},
$$

that is,

$$
z(s) \leq z(t)+r(t) z^{\prime}(t) \int_{t}^{s} \frac{d \theta}{r(\theta)}
$$

Because $r(t) z^{\prime}(t)$ is nonincreasing, we can find a constant $C>0$ such that $r(t) z^{\prime}(t) \leq$ $-C$ for $t \geq t_{2}$. As a result,

$$
z(s) \leq z(t)-C \int_{t}^{s} \frac{d \theta}{r(\theta)}
$$

and hence $0 \leq z(t)-C R_{1}(t)$ for $t \geq t_{2}$. Ultimately, $z\left(\tau_{k}\right) \geq C R_{1}\left(\tau_{k}\right), k \in \mathbb{N}$. From the system (2.2) it is easy to see that

$$
\begin{array}{r}
\left(r(t) z^{\prime}(t)\right)^{\prime}+q(t) G\left(C R_{1}(t-\sigma)\right) \leq 0, \quad t \neq \tau_{k} \\
\Delta\left(r\left(\tau_{k}\right) z^{\prime}\left(\tau_{k}\right)\right)+q\left(\tau_{k}\right) G\left(C R_{1}\left(\tau_{k}-\sigma\right)\right) \leq 0, \quad k \in \mathbb{N} .
\end{array}
$$


Integrating the last inequality from $t_{2}$ to $t\left(>t_{2}\right)$, we obtain

$$
\left[r(s) z^{\prime}(s)\right]_{t_{2}}^{t}+\int_{t_{2}}^{t} q(s) G\left(C R_{1}(s-\sigma)\right) d s-\sum_{t_{3} \leq \tau_{k}<t} \Delta\left(r\left(\tau_{k}\right) z^{\prime}\left(\tau_{k}\right)\right) \leq 0,
$$

that is,

$$
\begin{aligned}
\int_{t_{2}}^{t} q(s) G\left(C R_{1}(s-\sigma)\right) d s+\sum_{t_{2} \leq \tau_{k}<t} q_{k} G\left(C R_{1}\left(\tau_{k}-\sigma\right)\right) & \leq-\left[r(s) z^{\prime}(s)\right]_{t_{2}}^{t} \\
& \leq-r(t) z^{\prime}(t)
\end{aligned}
$$

implies that

$$
\frac{1}{r(t)}\left[\int_{t_{2}}^{t} q(s) G\left(C R_{1}(s-\sigma)\right) d s+\sum_{t_{2} \leq \tau_{k}<t} q_{k} G\left(C R_{1}\left(\tau_{k}-\sigma\right)\right)\right] \leq-z^{\prime}(t)
$$

and further integration of the preceding inequality, we have

$$
\begin{aligned}
\int_{t_{3}}^{u} \frac{1}{r(t)} & {\left[\int_{t_{3}}^{t} q(s) G\left(C R_{1}(s-\sigma)\right) d s+\sum_{t_{3} \leq \tau_{k}<t} q_{k} G\left(C R_{1}\left(\tau_{k}-\sigma\right)\right)\right] d t } \\
& \leq-[z(t)]_{t_{3}}^{u}+\sum_{t_{3} \leq \tau_{k}<u} \Delta z\left(\tau_{k}\right) \\
& =-[z(t)]_{t_{3}}^{u}+\sum_{t_{3} \leq \tau_{k}<u}\left[z\left(\tau_{k}+0\right)-z\left(\tau_{k}-0\right)\right] \\
& \leq z\left(t_{3}\right)+\sum_{t_{3} \leq \tau_{k}<u} z\left(\tau_{k}+0\right) \\
& <+\infty
\end{aligned}
$$

Ultimately,

$$
\int_{t_{3}}^{\infty} \frac{1}{r(t)}\left[\int_{t_{3}}^{t} q(s) G\left(C R_{1}(s-\sigma)\right) d s+\sum_{k=1}^{\infty} q_{k} G\left(C R_{1}\left(\tau_{k}-\sigma\right)\right)\right] d t<\infty
$$

gives a contradiction to $\left(A_{6}\right)$. Hence, $r(t) z^{\prime}(t)>0$ for $t \geq t_{2}$. As $z(t)$ is nondecreasing on $\left[t_{2}, \infty\right)$, there exist a constant $C>0$ and $t_{3}>t_{2}$ such that $z(t) \geq C$ for $t \geq t_{3}$. Therefore, the system (2.2) becomes

$$
\begin{array}{rr}
\left(r(t) z^{\prime}(t)\right)^{\prime}+q(t) G(C) \leq 0, \quad t \neq \tau_{k} \\
\Delta\left(r\left(\tau_{k}\right) z^{\prime}\left(\tau_{k}\right)\right)+q\left(\tau_{k}\right) G(C) \leq 0, \quad k \in \mathbb{N} .
\end{array}
$$

We integrate the preceding inequality from $t_{3}$ to $+\infty$ and obtain

$$
\int_{t_{3}}^{\infty} q(s) d s+\sum_{t_{3} \leq \tau_{k}<\infty} q\left(\tau_{k}\right)<+\infty
$$

which is a contradiction to $\left(A_{7}\right)$. Thus the proof of the theorem is complete.

Theorem 2.3. Let $-1<-a \leq p(t) \leq 0, a>0$ for $t \in \mathbb{R}_{+}$. Assume that $\left(A_{5}\right)$ and 
$\left(A_{8}\right) \int_{0}^{\infty} q(s) d s+\sum_{k=1}^{\infty} q\left(\tau_{k}\right) d t<\infty$

hold. Then the impulsive system $(E)$ admits a positive bounded solution.

Proof. Due to $\left(A_{5}\right)$, it is easy to verify that

$$
\int_{0}^{\infty} \frac{1}{r(s)}\left[\int_{s}^{\infty} q(t) d t+\sum_{k=1}^{\infty} q\left(\tau_{k}\right)\right] d s<+\infty .
$$

Let there exist $T>\rho$ such that

$$
G(R(t)) \int_{T}^{t} \frac{1}{r(s)}\left[\int_{s}^{\infty} q(\theta) d \theta+\sum_{k=1}^{\infty} q\left(\tau_{k}\right)\right] d s \leq \frac{R(t)}{4}, T \geq \rho .
$$

Consider

$$
\begin{array}{r}
M=\left\{y \in C([T-\sigma,+\infty), \mathbb{R}): y(t)=\frac{R(t)}{4}, t \in[T-\rho, T] ;\right. \\
\left.\frac{R(t)}{4} \leq y(t) \leq R(t) \text { for } t \geq T\right\}
\end{array}
$$

and let $\Phi: M \rightarrow M$ be defined by

$$
(\Phi y)(t)=\left\{\begin{array}{l}
(\Phi y)(T), \quad t-\rho \leq t \leq T \\
-p(t) y(t-\tau)+\frac{R(t)}{4}+\int_{T}^{t} \frac{1}{r(s)}\left[\int_{s}^{\infty} q(\theta) G(y(\theta-\sigma)) d \theta\right. \\
\left.+\sum_{k=1}^{\infty} q\left(\tau_{k}\right) G\left(y\left(\tau_{k}-\sigma\right)\right)\right] d s, \quad t \geq T .
\end{array}\right.
$$

For every $y \in M,(\Phi y)(t) \geq \frac{R(t)}{4}$ and

$$
\begin{aligned}
(\Phi y)(t) & \leq a R(t)+\frac{R(t)}{4}+G(R(t)) \int_{T}^{t} \frac{1}{r(s)}\left[\int_{s}^{\infty} q(\theta) d \theta+\sum_{k=1}^{\infty} q\left(\tau_{k}\right)\right] d s \\
& \leq a R(t)+\frac{R(t)}{4}+\frac{R(t)}{4}=\left(a+\frac{1}{2}\right) R(t) \leq R(t)
\end{aligned}
$$

implies that $(\Phi y) \in M$. Proceeding as in the proof of Theorem 2.1, we conclude that the operator $T$ has a fixed point $u \in M$, that is, $u(t)=(T u)(t), t \geq T-\rho$. Therefore, $u(t)$ is a solution of the impulsive system $(E)$ with $\frac{R(t)}{4} \leq u(t) \leq R(t)$ for $t \geq T$ which is regular and does not tend to zero as $t \rightarrow \infty$ when the limit exists. Thus the theorem is proved.

Theorem 2.4. Let $0 \leq p(t) \leq a<\infty$ for $t \in \mathbb{R}_{+}$. Assume that $\left(A_{1}\right)-\left(A_{3}\right)$ and $\left(A_{5}\right)$ hold. Furthermore, assume that

$\left(A_{9}\right)$ there exists $\lambda>0$ such that $G(u)+G(v) \geq \lambda G(u+v)$ for $u, v \in \mathbb{R}_{+}$,

$\left(A_{10}\right) G(u v) \leq G(u) G(v), u, v \in \mathbb{R}_{+}$,

$\left(A_{11}\right) \int_{T}^{\infty} \frac{1}{r(t)}\left[\int_{T_{1}}^{t} Q(s) G\left(C R_{1}(s-\sigma)\right) d s+\sum_{k=1}^{\infty} Q\left(\tau_{k}\right) G\left(C R_{1}\left(\tau_{k}-\sigma\right)\right)\right] d t$ $=+\infty, T, T_{1}>0$

and

$\left(A_{12}\right) \int_{T}^{\infty} Q(t) d t+\sum_{k=1}^{\infty} Q\left(\tau_{k}\right)=+\infty, T>\rho$

hold, where $Q(t)=\min \{q(t), q(t-\tau)\}, t \geq \tau$. Then every solution of the impulsive system $(E)$ oscillates. 
Proof. On the contrary, let $y(t)$ be a regular nonoscillatory solution of $(E)$. Proceeding as in Theorem 2.1, we have two cases namely $z(t)>0, r(t) z^{\prime}(t)<0$ and $z(t)>0$, $r(t) z^{\prime}(t)>0$ for $t \in\left[t_{2}, \infty\right)$. Consider the former one. Ultimately, $y(t)$ is bounded. Using the same type of argument as in the proof of the Theorem 2.2, we obtain that $z(t) \geq C R_{1}(t)$ for $t \geq t_{2}$. From the system $(E)$ it is easy to see that

$$
\begin{aligned}
& \quad\left(r(t) z^{\prime}(t)\right)^{\prime}+q(t) G(y(t-\sigma)) \\
& +G(a)\left[\left(r(t-\tau) z^{\prime}(t-\tau)\right)^{\prime}+q(t-\tau) G(y(t-\tau-\sigma))\right]=0, t \neq \tau_{k}, \\
& \Delta\left(r\left(\tau_{k}\right) z^{\prime}\left(\tau_{k}\right)\right)+q\left(\tau_{k}\right) G\left(y\left(\tau_{k}-\sigma\right)\right) \\
& +G(a)\left[\Delta\left(r\left(\tau_{k}-\tau\right) z^{\prime}\left(\tau_{k}-\tau\right)\right)+q\left(\tau_{k}-\tau\right) G\left(y\left(\tau_{k}-\tau-\sigma\right)\right)\right]=0, k \in \mathbb{N} .
\end{aligned}
$$

Using $\left(A_{9}\right)$ and $\left(A_{10}\right)$ in the above system, it follows that

$$
\begin{array}{r}
\left(r(t) z^{\prime}(t)\right)^{\prime}+G(a)\left(r(t-\tau) z^{\prime}(t-\tau)\right)^{\prime}+\lambda Q(t) G(z(t-\sigma)) \leq 0 \\
\Delta\left(r\left(\tau_{k}\right) z^{\prime}\left(\tau_{k}\right)\right)+G(a) \Delta\left(r\left(\tau_{k}-\tau\right) z^{\prime}\left(\tau_{k}-\tau\right)\right)+\lambda Q\left(\tau_{k}\right) G\left(z\left(\tau_{k}-\sigma\right)\right) \leq 0,
\end{array}
$$

where $z(t) \leq y(t)+a y(t-\tau)$. Ultimately, (2.4) reduces to

$$
\begin{aligned}
\left(r(t) z^{\prime}(t)\right)^{\prime}+G(a)\left(r(t-\tau) z^{\prime}(t-\tau)\right)^{\prime}+\lambda Q(t) G\left(C R_{1}(t-\sigma)\right) & \leq 0 \\
\Delta\left(r\left(\tau_{k}\right) z^{\prime}\left(\tau_{k}\right)\right)+G(a) \Delta\left(r\left(\tau_{k}-\tau\right) z^{\prime}\left(\tau_{k}-\tau\right)\right)+\lambda Q\left(\tau_{k}\right) G\left(C R_{1}\left(\tau_{k}-\sigma\right)\right) & \leq 0
\end{aligned}
$$

for $t \geq t_{3}>t_{2}, t \neq \tau_{k}, k \in \mathbb{N}$. Integrating the last system from $t_{3}$ to $t\left(>t_{3}\right)$, we get

$$
\begin{array}{r}
{\left[r(s) z^{\prime}(s)\right]_{t_{3}}^{t}+G(a)\left[r(s-\tau) z^{\prime}(s-\tau)\right]_{t_{3}}^{t}-\sum_{t_{3} \leq \tau_{k}<t} \Delta\left(r\left(\tau_{k}\right) z^{\prime}\left(\tau_{k}\right)\right)} \\
-G(a) \sum_{t_{3} \leq \tau_{k}<t} \Delta\left(r\left(\tau_{k}-\tau\right) z^{\prime}\left(\tau_{k}-\tau\right)\right)+\lambda \int_{t_{3}}^{t} Q(s) G\left(C R_{1}(s-\sigma)\right) d s \leq 0,
\end{array}
$$

that is,

$$
\begin{aligned}
\lambda\left[\int_{t_{3}}^{t} Q(s) G\left(C R_{1}(s-\sigma)\right) d s\right. & \left.+\sum_{t_{3} \leq \tau_{k}<t} Q\left(\tau_{k}\right) G\left(C R_{1}\left(\tau_{k}-\sigma\right)\right)\right] \\
& \leq-\left[r(s) z^{\prime}(s)+G(a)\left(r(s-\tau) z^{\prime}(s-\tau)\right)\right]_{t_{3}}^{t} \\
& \leq-\left[r(t) z^{\prime}(t)+G(a)\left(r(t-\tau) z^{\prime}(t-\tau)\right)\right] \\
& \leq-(1+G(a)) r(t) z^{\prime}(t) .
\end{aligned}
$$

Therefore,

$$
\frac{\lambda}{1+G(a)} \frac{1}{r(t)}\left[\int_{t_{3}}^{t} Q(s) G\left(C R_{1}(s-\sigma)\right) d s+\sum_{t_{3} \leq \tau_{k}<t} Q\left(\tau_{k}\right) G\left(C R_{1}\left(\tau_{k}-\sigma\right)\right)\right] \leq-z^{\prime}(t) .
$$

Integrating the above inequality, we obtain

$$
\frac{\lambda}{1+G(a)} \int_{t_{3}}^{\infty} \frac{1}{r(t)}\left[\int_{t_{3}}^{t} Q(s) G\left(C R_{1}(s-\sigma)\right) d s+\sum_{t_{3} \leq \tau_{k}<t} Q_{k} G\left(C R_{1}\left(\tau_{k}-\sigma\right)\right)\right] d t<\infty
$$


which is a contradiction to $\left(A_{11}\right)$. If the latter case holds, then there exist a constant $C>0$ and $t_{3}>t_{2}$ such that $z(t) \geq C$ for $t \geq t_{3}$. From (2.4), it follows that

$$
\begin{array}{r}
\left(r(t) z^{\prime}(t)\right)^{\prime}+G(a)\left(r(t-\tau) z^{\prime}(t-\tau)\right)^{\prime}+\lambda Q(t) G(C) \leq 0 \\
\Delta\left(r\left(\tau_{k}\right) z^{\prime}\left(\tau_{k}\right)\right)+G(a) \Delta\left(r\left(\tau_{k}-\tau\right) z^{\prime}\left(\tau_{k}-\tau\right)\right)+\lambda Q\left(\tau_{k}\right) G(C) \leq 0 .
\end{array}
$$

Integrating the last inequality from $t_{3}$ to $+\infty$, we get a contradiction to $\left(A_{12}\right)$. This completes the proof of the theorem.

Theorem 2.5. Let $0 \leq p(t) \leq R(t)<1$ for $t \in \mathbb{R}_{+}$. Assume that $\left(A_{5}\right)$ and $\left(A_{8}\right)$ hold. Furthermore, assume that $G$ is Lipschitzian on the intervals of the form $[a, b]$, $0<a<b<\infty$. Then the impulsive system $(E)$ admits a positive bounded solution.

Proof. Proceeding as in the proof of Theorem 2.3, we get (2.3). So, there exists $T>\rho$ such that

$$
\int_{T}^{\infty} \frac{1}{r(s)}\left[\int_{s}^{\infty} q(t) d t+\sum_{k=1}^{\infty} q\left(\tau_{k}\right)\right] d s<\frac{1-R(t)}{3 L}
$$

where $L=\max \left\{L_{1}, G(1)\right\}, L_{1}$ is the Lipschitz constant of $G$ on $\left[\frac{1-R(t)}{2}, 1\right]$ for $t \geq T$. Let $X=B C([T, \infty), \mathbb{R})$ be the space of real valued continuous functions on $[T, \infty)$. Indeed, $X$ is a Banach space with respect to the sup norm defined by

$$
\|y\|=\sup \{|y(t)|: t \geq T\} .
$$

Define

$$
S=\left\{v \in X: \frac{1-R(t)}{2} \leq v(t) \leq 1, t \geq T\right\} .
$$

We notice that $S$ is a closed and convex subspace of $X$. Let $\Phi: S \rightarrow S$ be such that

$$
(\Phi y)(t)=\left\{\begin{array}{l}
(\Phi y)(T+\rho), \quad t \in[T, T+\rho] \\
-p(t) y(t-\tau)+\frac{5+R(t)}{6}-\int_{t}^{\infty} \frac{1}{r(s)}\left[\int_{s}^{\infty} q(u) G(y(u-\sigma)) d u\right. \\
\left.+\sum_{k=1}^{\infty} q\left(\tau_{k}\right) G\left(y\left(\tau_{k}-\sigma\right)\right)\right] d s, t \geq T+\rho .
\end{array}\right.
$$

For every $y \in X,(\Phi y)(t) \leq \frac{5+R(t)}{6}<1$ and

$$
(\Phi y)(t) \geq-R(t)+\frac{5+R(t)}{6}-\frac{1-R(t)}{3}=\frac{1}{2}(1-R(t))
$$

implies that $\Phi y \in S$. For $y_{1}, y_{2} \in S$,

$$
\begin{aligned}
\left|\left(\Phi y_{1}\right)(t)-\left(\Phi y_{2}\right)(t)\right| & \leq R(t)\left|y_{1}(t-\tau)-y_{2}(t-\tau)\right| \\
& +\int_{t}^{\infty} \frac{1}{r(s)}\left[\int_{s}^{\infty} q(u)\left|G\left(y_{1}(u-\sigma)\right)-G\left(y_{2}(u-\sigma)\right)\right| d u\right. \\
& \left.+\sum_{k=1}^{\infty} q_{k}\left|G\left(y_{1}\left(\tau_{k}-\sigma\right)\right)-G\left(y_{2}\left(\tau_{k}-\sigma\right)\right)\right|\right] d s,
\end{aligned}
$$


that is,

$$
\begin{aligned}
\left|\left(\Phi y_{1}\right)(t)-\left(\Phi y_{2}\right)(t)\right| & \leq R(t)\left\|y_{1}-y_{2}\right\|+\left\|y_{1}-y_{2}\right\| L_{1} \\
& \times \int_{t}^{\infty} \frac{1}{r(s)}\left[\int_{s}^{\infty} q(u) d u+\sum_{k=1}^{\infty} q_{k}\right] d s \\
& \leq\left(R(t)+\frac{1-R(t)}{3}\right)\left\|y_{1}-y_{2}\right\|
\end{aligned}
$$

implies that

$$
\left|\left(\Phi y_{1}\right)(t)-\left(\Phi y_{2}\right)(t)\right| \leq \mu\left\|y_{1}-y_{2}\right\|
$$

where

$$
\left(R(t)+\frac{1-R(t)}{3}\right) \leq \frac{1+2 \alpha}{3}=\mu<1
$$

and $\alpha=\limsup \sup _{t \rightarrow \infty} R(t)\left(\because R(t)<\infty, R^{\prime}(t)>0\right)$. Therefore, $\Phi$ is a contraction. Using Banach's fixed point theorem, it follows that $\Phi$ has a unique fixed point $y(t)$ in $\left[\frac{1-R(t)}{2}, 1\right]$. This completes the proof of the theorem.

Theorem 2.6. Let $1<a_{1} \leq p(t) \leq a_{2}<\infty$, $a_{1}^{2} \geq a_{2}$ for $t \in \mathbb{R}_{+}$. Assume that $\left(A_{5}\right)$ and $\left(A_{8}\right)$ hold. Let $G$ be Lipschitzian on intervals of the form $[a, b], 0<a<b<\infty$. Then the impulsive system $(E)$ admits a positive bounded solution.

Proof. Proceeding as in the proof of Theorem 2.3, we have obtained (2.3). Let

$$
\int_{T}^{\infty} \frac{1}{r(t)}\left[\int_{t}^{\infty} q(s) d s+\sum_{k=1}^{\infty} q\left(\tau_{k}\right)\right] d t<\frac{a_{1}-1}{4 L},
$$

where $L=\max \left\{L_{1}, L_{2}\right\}, L_{1}$ is the Lipschitz constant of $G$ on $[a, b], L_{2}=G(b)$ with

$$
\begin{gathered}
a=\frac{4 \mu\left(a_{1}^{2}-a_{2}\right)-a_{2}\left(a_{1}-1\right)}{4 a_{1}^{2} a_{2}} \\
b=\frac{a_{1}-1+4 \mu}{4 a_{1}}, \quad \mu>\frac{a_{2}\left(a_{1}-1\right)}{4\left(a_{1}^{2}-a_{2}\right)}>0 .
\end{gathered}
$$

Let $X=B C([T, \infty), \mathbb{R})$ be the space of real valued functions defined on $[T, \infty)$. Indeed, $X$ is a Banach space with respect to sup norm defined by

$$
\|y\|=\sup \{|y(t)|: t \geq T\} .
$$

Define

$$
S=\{u \in X: a \leq u(t) \leq b, t \geq T\} .
$$

Let $\Phi: S \rightarrow S$ be such that

$$
(\Phi y)(t)=\left\{\begin{array}{l}
\Phi y(T+\rho), \quad t \in[T, T+\rho] \\
-\frac{y(t+\tau)}{p(t+\tau)}+\frac{\mu}{p(t+\tau)}+\frac{1}{p(t+\tau)} \int_{T}^{t+\tau} \frac{1}{r(s)}\left[\int_{s}^{\infty} q(v) G(y(v-\sigma)) d v\right. \\
\left.+\sum_{k=1}^{\infty} q\left(\tau_{k}\right) G\left(y\left(\tau_{k}-\sigma\right)\right)\right] d s, \quad t \geq T+\rho .
\end{array}\right.
$$


For every $y \in S$,

$$
\begin{aligned}
(\Phi y)(t) & \leq \frac{G(b)}{p(t+\tau)} \int_{T}^{t+\tau} \frac{1}{r(s)}\left[\int_{s}^{\infty} q(v) d v+\sum_{k=1}^{\infty} q\left(\tau_{k}\right)\right] d s+\frac{\mu}{p(t+\tau)} \\
& \leq \frac{G(b)}{p(t+\tau)} \int_{T}^{\infty} \frac{1}{r(s)}\left[\int_{s}^{\infty} q(v) d v+\sum_{k=1}^{\infty} q\left(\tau_{k}\right)\right] d s+\frac{\mu}{p(t+\tau)} \\
& \leq \frac{1}{a_{1}}\left[\frac{a_{1}-1}{4}+\mu\right]=b
\end{aligned}
$$

and

$$
(\Phi y)(t) \geq-\frac{y(t+\tau)}{p(t+\tau)}+\frac{\mu}{p(t+\tau)}>-\frac{b}{a_{1}}+\frac{\mu}{a_{2}}=a
$$

implies that $\Phi y \in S$. For $y_{1}, y_{2} \in S$

$$
\begin{aligned}
\left|\left(\Phi y_{1}\right)(t)-\left(\Phi y_{2}\right)(t)\right| & \leq \frac{1}{|p(t+\tau)|}\left|y_{1}(t+\tau)-y_{2}(t+\tau)\right| \\
& +\frac{G(b)}{|p(t+\tau)|} \int_{T}^{t+\tau} \frac{1}{r(s)}\left[\int_{s}^{\infty} q(v)\left|y_{1}(v-\sigma)-y_{2}(v-\sigma)\right| d v\right. \\
& \left.+\sum_{k=1}^{\infty} q\left(\tau_{k}\right)\left|y_{1}\left(\tau_{k}-\sigma\right)-y_{2}\left(\tau_{k}-\sigma\right)\right|\right] d s,
\end{aligned}
$$

that is,

$$
\begin{aligned}
\left|\left(\Phi y_{1}\right)(t)-\left(\Phi y_{2}\right)(t)\right| & \leq \frac{1}{a_{1}}\left\|y_{1}-y_{2}\right\|+\frac{G(b)}{a_{1}}\left\|y_{1}-y_{2}\right\| \\
& \times \int_{T}^{t+\tau} \frac{1}{r(s)}\left[\int_{s}^{\infty} q(v) d v+\sum_{k=1}^{\infty} q\left(\tau_{k}\right)\right] d s \\
& <\frac{1}{a_{1}}\left\|y_{1}-y_{2}\right\|\left(1+\frac{a_{1}-1}{4}\right) .
\end{aligned}
$$

Therefore,

$$
\left\|\left(\Phi y_{1}\right)-\left(\Phi y_{2}\right)\right\| \leq\left(\frac{1}{a_{1}}+\frac{a_{1}-1}{4 a_{1}}\right)\left\|y_{1}-y_{2}\right\| .
$$

As $\left(\frac{1}{a_{1}}+\frac{a_{1}-1}{4 a_{1}}\right)<1, \Phi$ is a contraction mapping. We note that $S$ is a closed convex subset of $X$ and hence by the Banach's fixed point theorem $\Phi$ has a unique fixed point, that is, $\Phi y(t)=y(t)$ on $[a, b]$. Thus the proof of the theorem is complete.

Theorem 2.7. Let $-\infty<-a_{1} \leq p(t) \leq-a_{2}<-1$ for $t \in \mathbb{R}_{+}$, where $a_{1}, a_{2}>0$. Assume that $\left(A_{1}\right)-\left(A_{3}\right)$ and $\left(\overline{A_{5}}\right)-\left(\overline{A_{7}}\right)$ hold. If

$\left(A_{13}\right) \int_{T}^{\infty} \frac{1}{r(t)}\left[\int_{T}^{t} q(s) d s+\sum_{k=1}^{\infty} q\left(\tau_{k}\right)\right] d t=+\infty$

then every bounded solution of the system $(E)$ either oscillates or converges to zero. 
Proof. Let $y(t)$ be a bounded regular solution of $(E)$. Proceeding as in Theorem 2.1, it follows that $z(t)$ and $r(t) z^{\prime}(t)$ are monotonic functions on $\left[t_{2}, \infty\right)$. Since $y(t)$ is bounded, then $z(t)$ is bounded and hence $\lim _{t \rightarrow \infty} z(t)$ exists. Using the arguments as in the proof of Theorem 2.2, we get contradictions to $\left(A_{6}\right)$ and $\left(A_{7}\right)$ for the cases $z(t)>0, r(t) z^{\prime}(t)<0$ and $z(t)>0, r(t) z^{\prime}(t)>0$ respectively. Consider the case $z(t)<0, r(t) z^{\prime}(t)>0$ for $t \geq t_{2}$. We claim that $\lim _{t \rightarrow \infty} z(t)=0$. If not, there exist $\beta<0$ and $t_{3}>t_{2}$ such that $z(t+\tau-\sigma)<\beta$ for $t \geq t_{3}$. Hence, $z(t) \geq-a_{1} y(t-\tau)$ implies that $y(t-\sigma) \geq-a_{1}^{-1} \beta$ for $t \geq t_{3}$. Consequently, the impulsive system (2.2) reduces to

$$
\begin{aligned}
\left(r(t) z^{\prime}(t)\right)^{\prime}+G\left(-a_{1}^{-1} \beta\right) q(t) \leq 0, & t \neq \tau_{k} \\
\Delta\left(r\left(\tau_{k}\right) z^{\prime}\left(\tau_{k}\right)\right)+G\left(-a_{1}^{-1} \beta\right) q\left(\tau_{k}\right) \leq 0, & k \in \mathbb{N}
\end{aligned}
$$

for $t \geq t_{3}$. Integrating (2.5) from $t_{3}$ to $+\infty$, we get

$$
\left[\int_{t_{3}}^{\infty} q(s) d s+\sum_{t_{3} \leq \tau_{k} \leq \infty} q\left(\tau_{k}\right)\right]<\infty
$$

which is a contradiction to $\left(A_{7}\right)$. So, our claim holds and

$$
\begin{aligned}
0 & =\lim _{t \rightarrow \infty} z(t)=\liminf _{t \rightarrow \infty}(y(t)+p(t) y(t-\tau)) \\
& \leq \liminf _{t \rightarrow \infty}\left(y(t)-a_{2} y(t-\tau)\right) \\
& \leq \limsup _{t \rightarrow \infty} y(t)+\liminf _{t \rightarrow \infty}\left(-a_{2} y(t-\tau)\right) \\
& =\left(1-a_{2}\right) \limsup _{t \rightarrow \infty} y(t)
\end{aligned}
$$

implies that $\limsup _{t \rightarrow \infty} y(t)=0 \quad\left[\because 1-a_{2}<0\right]$. Thus, $\lim _{t \rightarrow \infty} y(t)=0$.

Let $z(t)<0, r(t) z^{\prime}(t)<0$ for $t \geq t_{2}$. Proceeding as in the previous case, we get $(2.5)$. Integrating (2.5) from $t_{3}$ to $t$, we obtain

$$
\int_{t_{3}}^{t} q(s) G\left(-a_{1}^{-1} \beta\right) d s+\sum_{t_{3} \leq \tau_{k} \leq t} q\left(\tau_{k}\right) G\left(-a_{1}^{-1} \beta\right) \leq-r(t) z^{\prime}(t),
$$

that is,

$$
\frac{1}{r(t)}\left[\int_{t_{3}}^{t} q(s) G\left(-a_{1}^{-1} \beta\right) d s+\sum_{t_{3} \leq \tau_{k} \leq t} q\left(\tau_{k}\right) G\left(-a_{1}^{-1} \beta\right)\right] \leq-z^{\prime}(t)
$$

for $t \geq t_{3}$. Further integration of the above inequality from $t_{3}$ to $+\infty$, we get

$$
\int_{t_{3}}^{\infty} \frac{1}{r(t)}\left[\int_{t_{3}}^{t} q(s) d s+\sum_{t_{3} \leq \tau_{k} \leq t} q\left(\tau_{k}\right)\right] d t<\infty
$$

which contradicts $\left(A_{13}\right)$. Thus $\lim _{t \rightarrow \infty} z(t)=0$. Rest of this case follows from the previous case. This completes the proof of the theorem.

Theorem 2.8. Let $-\infty<-a_{1} \leq p(t) \leq-a_{2}<-1$ for $t \in \mathbb{R}_{+}$, where $a_{1}, a_{2}>0$ such that $4 a_{2}>a_{1}$. Assume that $\left(A_{5}\right)$ and $\left(A_{8}\right)$ hold. Furthermore, assume that $G$ is Lipschitzian on the intervals of the form $[a, b], 0<a<b<\infty$. Then the system $(E)$ admit a positive bounded solution. 
Proof. Proceeding as in the proof of Theorem 2.3, we get (2.3). So, it is possible to find $T>\rho$ such that

$$
\int_{T}^{\infty} \frac{1}{r(s)}\left[\int_{s}^{\infty} q(t) d t+\sum_{k=1}^{\infty} q\left(\tau_{k}\right)\right] d s<\frac{a_{2}-1}{4 L},
$$

where $L=\max \left\{L_{1}, G(1)\right\}, L_{1}$ is the Lipschitz constant of $G$ on $(a, 1)$,

$$
a=\frac{\left(a_{2}-1\right)\left(4 a_{2}-a_{1}\right)}{4 a_{1} a_{2}} .
$$

Let $X=B C([T, \infty), \mathbb{R})$ be the space of real valued continuous functions defined on $[T, \infty)$. Indeed, $X$ is a Banach space with the supremum norm defined by

$$
\|y\|=\sup \{|y(t)|: t \geq T\}
$$

Define

$$
S=\{v \in X: a \leq v(t) \leq 1, t \geq T\} .
$$

We may note that $S$ is a closed and convex subspace of $X$. Let $\Psi: S \rightarrow S$ be such that

$$
(\Psi y)(t)=\left\{\begin{array}{l}
\Psi y(T+\rho), \quad t \in[T, T+\rho] \\
-\frac{y(t+\tau)}{p(t+\tau)}-\frac{a_{2}-1}{p(t+\tau)}+\frac{1}{p(t+\tau)} \int_{T}^{t+\tau} \frac{1}{r(s)}\left[\int_{s}^{\infty} q(u) G(y(u-\sigma)) d u\right. \\
\left.+\sum_{k=1}^{\infty} q\left(\tau_{k}\right) G\left(y\left(\tau_{k}-\sigma\right)\right)\right] d s, \quad t \geq T+\rho .
\end{array}\right.
$$

For every $y \in S$,

$$
\begin{aligned}
(\Psi y)(t) & \leq-\frac{y(t+\tau)}{p(t+\tau)}-\frac{a_{2}-1}{p(t+\tau)} \\
& \leq \frac{1}{a_{2}}+\frac{a_{2}-1}{a_{2}}=1
\end{aligned}
$$

and

$$
\begin{aligned}
(\Psi y)(t) & \geq-\frac{a_{2}-1}{p(t+\tau)}+\frac{1}{p(t+\tau)} \\
& \times \int_{T}^{t+\tau} \frac{1}{r(s)}\left[\int_{s}^{\infty} q(u) G(y(u-\sigma)) d u+\sum_{k=1}^{\infty} q\left(\tau_{k}\right) G\left(y\left(\tau_{k}-\sigma\right)\right)\right] d s \\
& \geq \frac{a_{2}-1}{a_{1}}+\frac{G(1)}{p(t+\tau)} \int_{T}^{t+\tau} \frac{1}{r(s)}\left[\int_{s}^{\infty} q(u) d u+\sum_{k=1}^{\infty} q\left(\tau_{k}\right)\right] d s \\
& \geq \frac{a_{2}-1}{a_{1}}-\frac{G(1)}{a_{2}} \int_{T}^{\infty} \frac{1}{r(s)}\left[\int_{s}^{\infty} q(u) d u+\sum_{k=1}^{\infty} q\left(\tau_{k}\right)\right] d s \\
& \geq \frac{a_{2}-1}{a_{1}}-\frac{a_{2}-1}{4 a_{2}}=a
\end{aligned}
$$


implies that $(\Psi y) \in S$. For $y_{1}, y_{2} \in S$, we have that

$$
\begin{aligned}
\left|\left(\Psi y_{1}\right)(t)-\left(\Psi y_{2}\right)(t)\right| & \leq \frac{1}{|p(t+\tau)|}\left|y_{1}(t+\tau)-y_{2}(t+\tau)\right| \\
& +\frac{L_{1}}{|p(t+\tau)|} \int_{T}^{t+\tau} \frac{1}{r(s)}\left[\int_{s}^{\infty} q(u)\left|y_{1}(u-\sigma)-y_{2}(u-\sigma)\right| d u\right. \\
& \left.+\sum_{k=1}^{\infty} q\left(\tau_{k}\right)\left|y_{1}\left(\tau_{k}-\sigma\right)-y_{2}\left(\tau_{k}-\sigma\right)\right|\right] d s,
\end{aligned}
$$

that is,

implies that

$$
\left|\left(\Psi y_{1}\right)(t)-\left(\Phi y_{2}\right)(t)\right| \leq \frac{1}{a_{2}}\left\|y_{1}-y_{2}\right\|+\frac{a_{2}-1}{4 a_{2}}\left\|y_{1}-y_{2}\right\|
$$

$$
\left\|\left(\Psi y_{1}\right)-\left(\Psi y_{2}\right)\right\| \leq \mu\left\|y_{1}-y_{2}\right\|,
$$

where $\mu=\frac{1}{a_{2}}\left(1+\frac{a_{2}-1}{4}\right)<1$. Therefore, $\Psi$ is a contraction. By the Banach's fixed point theorem, $\Psi$ has a unique fixed point $y \in S$. It is easy to see that $\lim _{t \rightarrow \infty} y(t) \neq 0$. This completes the proof of the theorem.

\section{Discussion and example}

It is worth observation that we could succeed to establish the necessary and sufficient conditions for oscillation of all solutions of the impulsive system $\left(E_{1}\right)$ when $-1<p(t) \leq 0$ only. However, we failed to obtain the necessary and sufficient conditions for the other ranges of $p(t)$ and hence the undertaken problem is open for other ranges of $p(t)$. May be some other method is required to overcome the problem.

We conclude this section with the following example:

Example 3.1. Consider the impulsive system

$$
\left(E_{4}\right)\left\{\begin{array}{l}
\left(r(t)(y(t)+p(t) y(t-1))^{\prime}\right)^{\prime}+q(t) y(t-1)=0, t \neq \tau_{k} \\
\Delta\left(r\left(\tau_{k}\right)\left(y\left(\tau_{k}\right)+p\left(\tau_{k}\right) y\left(\tau_{k}-1\right)\right)^{\prime}\right)+q\left(\tau_{k}\right) y\left(\tau_{k}-1\right)=0, \quad k \in \mathbb{N},
\end{array}\right.
$$

where $-1<p(t)=e^{-t}-1 \leq 0, q(t)=e^{-t}, r(t)=e^{t}, R(t)=1-e^{-t}, G(x)=x$, $\rho=1$ and $\tau_{k}=2^{k}, k \in \mathbb{N}$. Clearly, all conditions of Theorem 2.1 are satisfied. Thus by Theorem 2.1 , every unbounded solution of the system $\left(E_{4}\right)$ oscillates.

\section{References}

[1] Bainov, D.D., Simeonov, P.S., Systems with Impulse Effect: Stability, Theory and Applications, Ellis Horwood, Chichester, 1989.

[2] Bainov, D.D., Simeonov, P.S., Theory of Impulsive Differential Equations: Asymptotic Properties of the Solutions and Applications, World Scientific Publishers, Singapore, 1995.

[3] Bainov, D.D., Dimitrova, M.B., Oscillation of sub and super linear impulsive differential equations with constant delay, Applicable Analysis., 64(1997), 57-67. 
[4] Bonotto, E.M., Gimenes, L.P., Federson, M., emphOscillation for a second order neutral differential equation with impulses, Appl. Math. Compu., 215(2009), 1-15.

[5] Dimitrova, M.B., Oscillation criteria for the solutions of nonlinear delay differential equations of second order with impulse effect, Int. J. Pure and Appl. Math., 72(2011), 439-451.

[6] Domoshnitsky, A., Landsman, G., Yanetz, S., About sign-constancy of green's functions for impulsive second order delay equations, Opuscula Math., 34(2)(2014), 339-362.

[7] Gimenes, L.P., Federson, M., Oscillation by impulses for a second order delay differential equations, Cadernos De Matematica., 6(2005), 181-191.

[8] Graef, J.R., Grammatikopoulos, M.K., On the behaviour of a first order nonlinear neutral delay differential equations, Applicable Anal., 40(1991), 111-121.

[9] Graef, J.R., Shen, J.H., Stavroulakis, I.P., Oscillation of impulsive neutral delay differential equations, J. Math. Anal. Appl., 268(2002), 310-333.

[10] Gyori, I., Ladas, G., Oscillation Theory of Delay Differential Equations with Applications, Clarendon Press, Oxford, 1991.

[11] He, Z.M., Ge, W., Oscillation in second order linear delay differential equation with nonlinear impulses, Mathematica Slovaca, 52(2002), 331-341.

[12] Jiang, G.R., Lu, Q.S., Impulsive state feedback control of a prey-predator model, J. Compu. Appl. Math., 200(2007), 193-207.

[13] Ladde, G.S., Lakshmikantham, V., Zhang, B.G., Oscillation Theory of Differential Equations with Deviating Arguments, Pure and Applied Mathematics., 110 1987, Marcel Dekker.

[14] Lakshmikantham, V., Bainov, D.D., Simieonov, P.S., Oscillation Theory of Impulsive Differential Equations, World Scientific, Singapore, 1989.

[15] Liu, X., Ballinger, G., Existence and continuability of solutions for differential equations with delays and state-dependent impulses, Nonlin. Anal., 51(2002), 633-647.

[16] Luo, W., Luo, J., Debnath, L., Oscillation of second order quasilinear delay differential equations with impulses, J. Appl. Math. Comp., 13(2003), 165-182.

[17] Luo, Z., Shen, J., Oscillation of second order linear differential equation with impulses, Appl. Math. Lett., 20(2007), 75-81.

[18] Pandit, S.G., Deo, S.G., Differential Systems Involving Impulses, Lect. Notes in Math., 954, Springer-Verlag, New York, 1982.

[19] Qian, L., Lu, Q., Meng, Q., Feng, Z., Dynamical behaviours of a prey-predator system with impulsive control, J. Math. Anal. Appl., 363(2010), 345-356.

[20] Santra, S.S., Oscillation criteria for nonlinear neutral differential equations of first order with several delays, Mathematica (Cluj), 57(80)(2015), no. 1-2, 75-89.

[21] Santra, S.S., Necessary and sufficient condition for oscillation of nonlinear neutral first order differential equations with several delays, Mathematica (Cluj), 58(81)(2016), no. 1-2, 85-94.

[22] Santra, S.S., Oscillation analysis for nonlinear neutral differential equations of second order with several delays, Mathematica (Cluj), 59(82)(2017), no. 1-2, 111-123.

[23] Santra, S.S., Oscillation analysis for nonlinear neutral differential equations of second order with several delays and forcing term, Mathematica (Cluj), 61(84)(2019), no. 1, 63-78. 
[24] Santra, S.S., Tripathy, A.K., Oscillation of unforced impulsive neutral delay differential equations of first order, Commu. Appl. Anal., 22(4)(2018), 567-582.

[25] Santra, S.S., Tripathy, A.K., On oscillatory first order nonlinear neutral differential equations with nonlinear impulses, J. Appl. Math. Comput. (DOI: https://doi.org/10.1007/s12190-018-1178-8) (in press).

[26] Shen, J., Liu, Y., Asymptotic behaviour of solutions for nonlinear delay differential equation with impulses, Appl. Math. Compu., 213(2009), 449-454.

[27] Thandapani, E., Sakthivel, R., Chandrasekaran, E., Oscillation of second order nonlinear impulsive differential equations with deviating arguments, Diff. Equ. Appl., 4(2012), 571580 .

[28] Tripathy, A.K., Oscillation criteria for a class of first order neutral impulsive differentialdifference equations, J. Appl. Anal. Compu., 4(2014), 89-101.

[29] Tripathy, A.K., Santra, S.S., Necessary and sufficient conditions for oscillation of a class of first order impulsive differential equations, Func. Diff. Equ., 22(2015), no. 3-4, 149-167.

[30] Tripathy, A.K., Santra, S.S., Pulsatile constant and characterisation of first order neutral impulsive differential equations, Commu. Appl. Anal., 20(2016), 65-76.

[31] Tripathy, A.K., Santra, S.S., Characterization of a class of second order neutral impulsive systems via pulsatile constant, Diff. Equ. Appl., 9(2017), no. 1, 87-98.

[32] Xiu-li, W., Si-yang, C., Hong-ji, T., Osillation of a class of second order delay differential equation with impulses, Appl. Math. Comp., 145(2003), 561-567.

[33] Xiu-li, W., Si-yang, C., Hong-ji, T., Osillation of a class of second order nonlinear ODE with impulses, Appl. Math. Comp., 138(2003), 181-188.

[34] Yan, J., Oscillation properties of a second order impulsive delay differential equations, Compu. Math. Appl., 47(2004), 253-258.

Arun Kumar Tripathy

Department of Mathematics, Sambalpur University,

Sambalpur - 768019, India

e-mail: arun_tripathy70@rediffmail.com

Shyam Sundar Santra

Department of Mathematics, JIS College of Engineering,

Kalyani - 741235, India

(Corresponding author)

e-mail: shyam01.math@gmail.com 